\title{
Dynamical mean-field approach with predictive power for strongly correlated materials
}

\author{
D. Vollhardt ${ }^{1, a}$ and A.I. Lichtenstein ${ }^{2, b}$ \\ 1 Theoretical Physics III, Center for Electronic Correlations and Magnetism, Institute of \\ Physics, University of Augsburg, 86135 Augsburg, Germany \\ 2 I. Institut für Theoretische Physik, Universität Hamburg, Jungiusstraße 9, \\ 20355 Hamburg, Germany
}

Received 20 April 2017

Published online 10 July 2017

\begin{abstract}
Electronic correlations in solids are known to lead to the emergence of novel, unexpected phenomena, which are not only of interest for fundamental research but also have a great potential for technological applications. Hence there is a great need for appropriate theoretical techniques that allow for an accurate exploration of correlated electron materials. For a long time first-principles investigations of correlated materials were out of reach. During that time the electronic properties of solids were investigated by two essentially separate communities, one employing density functional theory (DFT), the other studying model Hamiltonians using many-body techniques.

Here the Dynamical Mean-Field Theory (DMFT), whose development started more than 25 years ago, opened new perspectives. In contrast to single-particle theories the mean-field of the DMFT is energy dependent, i.e., dynamical. Thereby the local quantum fluctuations on the impurity due to the bath are fully taken into account. The only approximation of the DMFT is the neglect of spatial fluctuations. Thus DMFT provides a comprehensive theoretical framework for the investigation of correlated lattice models and can describe, for example, fluctuating moments, the renormalization of quasiparticles, and the correlation induced spectral-weight transfer between low- and high-energy states.

To go beyond model studies and investigate real materials with strongly correlated electrons, the combination of DFT in the local density approximation (LDA) with the many-body DMFT, the so-called "LDA+DMFT" approach, was initiated 20 years ago. Starting from band structure theory, local correlations are taken into account by interaction terms which can be parametrized in particular by the Hubbard $U$ and the Hund's rule coupling $J$. The resulting coupled, self-consistent LDA+DMFT equations have to be solved numerically, usually by employing quantum Monte-Carlo techniques.
\end{abstract}

\footnotetext{
a e-mail: dieter.vollhardt@physik.uni-augsburg.de

b e-mail: alexander.lichtenstein@physik.uni-hamburg.de
} 
LDA+DMFT is a many-body approach which is able to describe the physics of materials with correlated electrons for all strengths of the effective interaction and all dopings. It already proved to be remarkably successful in the investigation a large number of correlated materials, ranging from transition metals and their oxides, $f$-electron systems, and Heusler alloys, all the way to heterostructures of complex oxides and doped organic compounds. Nevertheless the LDA+DMFT scheme still needs to be improved in numerous ways. This was exactly the motivation behind the foundation of the Research Unit FOR 1346 Dynamical Mean-Field Approach with Predictive Power for Strongly Correlated Materials: namely, to bring together experts in electronic band theory, materials science, many-body approaches, quantum impurity solvers, and numerical optimization techniques to advance the dynamical mean-field approach into a comprehensive, versatile ab initio method for the investigation of correlated electron materials. Clearly, such a demanding goal can only be reached by a concerted research effort.

The Research Unit FOR 1346 was funded by the Deutsche Forschungsgemeinschaft from 2010 to 2017. It was the first coherent activity in this field world-wide. Its ultimate goal was to create a new standard of computational electronic structure scheme which is suitable to explore the properties of complex, correlated materials. From the very start it was clear that this is an extremely ambitious task, which would require years and the effort of the entire international community.

During the second funding period (2013-2017) the Research Unit consisted of ten projects, eight of which were theoretical and two were collaborations between experimentalists and theorists. It was initiated by 29 principal investigators from 17 institutions in Austria, Germany, and Switzerland, and involved a large number of researchers from all over the world, as evidenced by the author list of the articles of the present volume. For more detailed information we refer to the Research Unit's homepage:

http://www .physik. uni-augsburg.de/for1346/

The Research Unit FOR 1346 made great progress during its funding period. In particular, the joint effort of individual projects resulted in important developments regarding the design of efficient impurity solvers and the development of realistic DMFT codes, the investigation of structural transformations and phonons anomalies, the calculation of large-scale superlattices for Mott materials, the analysis of complex photoemission spectra of correlated materials, and the prediction of anomalous properties of correlated electron systems. Some of the most prominent achievements are reported and discussed in the eleven articles collected in this special issue of European Physical Journal - Special Topics. They are grouped according to the following four methodological challenges:

1. Electronic structure of paradigmatic correlated materials

2. Efficient quantum-impurity solvers for dynamical mean-field theory

3. Non-local interactions and spatial correlations

4. Ordering phenomena and lattice transformations

In the first three papers, part (1), some of our Research Unit's studies of the electronic structure of typical correlated materials are described: T. Haupricht et al. [1] report results of a detailed experimental and theoretical study of the electronic structure of $\mathrm{NiO}$. In this material the charge-transfer nature of the band gap and the intricate interplay between local electronic correlations and band formation lead to challenges of a quantitative ab-initio modeling of the electronic structure. Furthermore, the presence of non-local screening processes makes it necessary to go beyond 
single-site many-body approaches to explain the valence band spectrum. M. Sing et al. [2] discuss the influence of oxygen vacancies on two-dimensional electron systems at $\mathrm{SrTiO}_{3}$-based interfaces and surfaces. The insulator $\mathrm{SrTiO}_{3}$ can host high-mobility two-dimensional electron systems on its surfaces and at interfaces with other oxides. Based on calculations using DFT and DMFT as well as on experimental results using photoemission spectroscopy they elucidate the role of oxygen vacancies, thereby highlighting their importance for the electronic and magnetic properties of this system. J. Minár et al. [3] review the developments and applications of a combination of DFT and DMFT, the self-consistent LSDA+DMFT method, implemented within the fully relativistic KKR (Korringa-Kohn-Rostoker) band structure method. In particular, they discuss the spin-orbit induced orbital magnetic moments in $\mathrm{Fe}_{x} \mathrm{Ni}_{1-x}$ disordered alloys, the magnetic Compton profiles of fcc $\mathrm{Ni}$ and the angle-resolved photoemission spectroscopy (ARPES) spectra for gallium manganese arsenide dilute magnetic semiconductors.

The following three papers, part (2), address the development and application of efficient quantum-impurity solvers for DMFT: Shinaoka et al. [4] discuss Monte Carlo based impurity solvers. They present an efficient implementation of the hybridization expansion approach, which enables the simulation of multi-orbital impurity problems with off-diagonal and complex hybridizations, and dynamically screened (retarded) density-density interactions. As a complementary approach an impurity solver based on the determinant Monte Carlo method is discussed, which scales favorably with inverse temperature and hence provides access to the very low temperature regime. Baumgärtel et al. [5] report results of massively parallel simulations of strong electronic correlations. They show that both Lanczos and quantum Monte Carlo in different flavors exhibit excellent scaling on massively parallel supercomputers, and apply these algorithms to simulate realistic model Hamiltonians including the full Coulomb vertex, crystal-field splitting, and spin-orbit interaction. The authors show how to extract the physically observable quantities from imaginary time data, in particular correlation functions and susceptibilities, and they present benchmarks and applications for representative correlated systems. Yi Lu and Haverkort [6] use exact diagonalization as an impurity solver in DMFT. While most implementations solve the DMFT equations using quantum Monte-Carlo sampling on the imaginary time and frequency axis, these authors explore alternative methods using exact diagonalization, i.e., representing the many-body ground state of the impurity as a sum over Slater determinants and calculating Green's functions using iterative Lanczos procedures. Recent advances in exact diagonalization which can evade the exponential barrier are reviewed.

In part (3) the importance of non-local interactions and spatial correlations are explored. Held et al. [7] describe recent developments in electronic structure calculations that go beyond state-of-the-art methods such as DFT and DMFT. Specifically, they discuss the GW approximation as implemented in the Vienna ab-initio simulation package (VASP) with the self-energy on the imaginary frequency axis, the merging of GW with DMFT, and the ab-initio dynamical vertex approximation DГA. The latter includes the physics of GW, DMFT and non-local correlations beyond, and allows one to calculate (quantum) critical exponents. Lechermann et al. [8] discuss realistic many-body approaches to materials with strong non-local correlations. While local correlations are in principle treated exactly within DMFT, there are two major and interlinked routes for important further methodical advances: On one hand, there is a strong need for methods combining the DMFT with modern band-structure theory, and on the other hand non-local correlations beyond the mean-field paradigm must be accounted for. Referring to several concrete examples the authors discuss how these two routes can be combined. Schüler et al. [9] review method developments and applications of theoretical approaches for the realistic description of the electronic and 
magnetic properties of nanostructures with correlated electrons. The implementation of a flexible interface between DFT and a variant of DMFT suitable for the simulation of complex correlated structures is explained and illustrated. Discussing Cr (001) surfaces, magnetic adatoms, and molecular systems as examples it is shown how the interplay of the Hubbard $U$ and Hund's $J$ determines charge and spin fluctuations and how these interactions drive different sorts of correlation effects in nanosystems.

Finally, part (4) is devoted to lattice transformations and ordering phenomena in general. Kuneš et al. [10] discuss the search for electronic instabilities in materials close to spin-state crossovers and analyze the properties of the corresponding ordered states within DMFT. This investigation, motivated by the physics of $\mathrm{LaCoO}_{3}$, led to the discovery of the condensation of spinful excitons in the two-orbital Hubbard model, with a surprisingly rich phase diagram. Electronic correlations can also be the driving force behind structural transformations of materials. To be able to investigate such instabilities, a formalism for the computation of total energies and forces was developed within a fully charge self-consistent combination of DFT and DMFT. The properties of Fe and FeSe determined within this approach are discussed. Schade et al. [11] review the current state of reduced density-matrix functional theory, which is a natural extension of DFT for systems dominated by orbital physics. They describe a method which combines many-particle effects based on reduced densitymatrix functional theory with a density functional-like framework, and present the construction of density-matrix functionals directly from many-particle theory such as methods from quantum chemistry or many-particle Greens functions.

Results obtained within the Research Unit FOR 1346 can also be found in the lecture notes of the two Autumn Schools organized by us at the Forschungszentrum Jülich in 2011 and 2014, respectively:

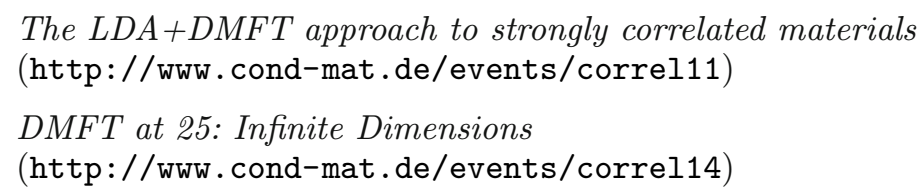

The basis for the success of the Research Unit FOR 1346 was the excellent cooperation between its numerous members - all experts in their respective field of research. In particular, the highly significant contributions by young researchers should be stressed.

All members of the Research Unit FOR 1346 are very grateful to the Deutsche Forschungsgemeinschaft for the funding during the last seven years which made this research possible.

This special issue of EPJ-ST is dedicated to the memory of our colleague Thomas Pruschke (1959-2016), one of the principal investigators of project P9, whose untimely death has been an immense loss not only for our Research Unit but for the entire correlated electron community.

Open Access This is an Open Access article distributed under the terms of the Creative Commons Attribution License (http://creativecommons.org/licenses/by/4.0), which permits unrestricted use, distribution, and reproduction in any medium, provided the original work is properly cited.

\section{References}

1. T. Haupricht, J. Weinen, A. Tanaka, M.W. Haverkort, L.H. Tjeng, Eur. Phys. J. Special Topics 226, 2245 (2017)

2. M. Sing, H.O. Jeschke, F. Lechermann, R. Valenti, R. Claessen, Eur. Phys. J. Special Topics 226, 2457 (2017) 
3. J. Minár, H. Ebert, L. Chioncel, Eur. Phys. J. Special Topics 226, 2477 (2017)

4. H. Shinaoka, P. Werner, F. Assaad, N. Blümer, Eur. Phys. J. Special Topics 226, 2499 (2017)

5. M. Baumgärtel, K.Ghanem, A. Kiani, E. Koch, E. Pavarini, E. Sarvestani, H. Sims, G. Zhang, Eur. Phys. J. Special Topics 226, 2525 (2017)

6. Y. Lu, M.W. Haverkort, Eur. Phys. J. Special Topics 226, 2549 (2017)

7. K. Held, G. Kresse, J. Tomczak, A. Toschi, Eur. Phys. J. Special Topics 226, 2565 (2017)

8. F. Lechermann, A.I. Lichtenstein, M. Potthoff, Eur. Phys. J. Special Topics 226, 2591 (2017)

9. M. Schüler, S. Barthel, T. Wehling, M. Karolak, A. Valli, G. Sangiovanni, Eur. Phys. J. Special Topics 226, 2615 (2017)

10. J. Kuneš, I. Leonov, P. Augustinský, V. Křápek, M. Kollar, D. Vollhardt, Eur. Phys. J. Special Topics 226, 2641 (2017)

11. R. Schade, E. Kamil, P. Bloechl, Eur. Phys. J. Special Topics 226, 2677 (2017) 